\title{
Total laparoscopic living donor right hepatectomy
}

\author{
Ho-Seong Han • Jai Young Cho $\cdot$ Yoo-Seok Yoon • \\ Dae Wook Hwang $\cdot$ Young Ki Kim • \\ Hong Kyung Shin · Woohyung Lee
}

Received: 5 December 2013/ Accepted: 26 May 2014/Published online: 4 July 2014

(C) Springer Science+Business Media New York 2014

\begin{abstract}
Background Right lobe living donor liver transplantation (LDLT) is the predominant form of adult-to-adult LDLT. Accordingly, cosmetic and functional demand by young donors is increasing. We developed the world first total laparoscopic donor right hepatectomy (LDRH) in adult living donors.

Methods Total LDRH was performed in two young donors without vascular clamping. Modified extended right graft (right liver including all the middle hepatic vein branches) was retrieved from suprapubic transverse incision.

Findings After full mobilization of right liver, hilar dissection was done. First, right portal vein was isolated under retracting common bile duct laterally. Right hepatic artery was cautiously dissected and isolated without injuring. An exact transection line was drawn during transient clamping of the hepatic artery and portal vein on the right side of the liver using bulldog clamp. Dissection was meticulously performed along the right side of the middle hepatic vein until the origin of middle hepatic vein until exposure of the hilar plate. Anterior section vein branches (V5 and V8) were finely dissected and were reconstructed using an artificial vascular graft. A modified extended right graft
\end{abstract}

This video was considered one of the best submissions to 2014 SAGES Annual Meeting.

Electronic supplementary material The online version of this article (doi:10.1007/s00464-014-3649-9) contains supplementary material, which is available to authorized users.

H.-S. Han · J. Y. Cho $(\bowtie) \cdot$ Y.-S. Yoon ·

D. W. Hwang · Y. K. Kim · H. K. Shin · W. Lee

Department of Surgery, Seoul National University Bundang

Hospital, Seoul National University College of Medicine, 300

Gumi-dong, Bundang-gu, Seongnam-si 463-707, Gyeonggi-do,

Republic of Korea

e-mail: jychogs@gmail.com with preservation of the middle hepatic vein branches was extracted through the suprapubic incision. There was no complication in both donors and recipients. Postoperative hospital stay of donors was 10 and 8 days, respectively. After follow-up of more than 1 year, all donors and recipients live well with normal liver function.

Conclusion Total LDRH was feasible in selected adult donors. If this procedure will be more standardized, then total LDRH will be new option for adult LDLT, which meets demand by donors and diminish guilty feeling by recipients [1-5].

Keywords Liver transplantation - Living donor - Right liver graft · Cosmesis

Disclosures Ho-Seong Han, Jai Young Cho, Yoo-Seok Yoon, Dae Wook Hwang, Young Ki Kim, Hong Kyung Shin and Woohyung Lee have no conflicts of interest.

\section{References}

1. Samstein B, Cherqui D, Rotellar F, Griesemer A, Halazun KJ, Kato T, Guarrera J, Emond JC (2013) Totally laparoscopic full left hepatectomy for living donor liver transplantation in adolescents and adults. Am J Transplant 13:2462-2466

2. Rotellar F, Pardo F, Benito A, Marti-Cruchaga P, Zozaya G, Lopez L, Hidalgo F, Sangro B, Herrero I (2013) Totally laparoscopic right-lobe hepatectomy for adult living donor liver transplantation: useful strategies to enhance safety. Am J Transplant 13:3269-3273

3. Soubrane O, Perdigao Cotta F, Scatton O (2013) Pure laparoscopic right hepatectomy in a living donor. Am J Transplant 13:2467-2471

4. Kim KH, Jung DH, Park KM, Lee YJ, Kim DY, Kim KM, Lee SG (2011) Comparison of open and laparoscopic live donor left lateral sectionectomy. Br J Surg 98:1302-1308

5. Cherqui D, Soubrane O, Husson E, Barshasz E, Vignaux O, Ghimouz M, Branchereau S, Chardot C, Gauthier F, Fagniez PL, Houssin D (2002) Laparoscopic living donor hepatectomy for liver transplantation in children. Lancet 359:392-396 